\title{
Transcription of the human uncoupling protein 3 gene is governed by a complex interplay between the promoter and intronic sequences
}

\author{
A. Girousse • G. Tavernier • C. Tiraby • \\ L. Lichtenstein • J. S. Iacovoni • A. Mairal • \\ F. Villarroya $\cdot$ D. Langin
}

Received: 23 February 2009 / Accepted: 17 April 2009 /Published online: 26 May 2009

(C) Springer-Verlag 2009

\begin{abstract}
Aims/hypothesis Uncoupling protein (UCP) 3 is an inner mitochondrial membrane transporter mainly produced in skeletal muscle in humans. UCP3 plays a role in fatty acid metabolism and energy homeostasis and modulates insulin sensitivity. In humans, UCP3 content is higher in fasttwitch glycolytic muscle than in slow-twitch oxidative muscle and is dysregulated in type 2 diabetes. Here, we
\end{abstract}

A. Girousse $\cdot$ G. Tavernier $(\bowtie) \cdot$ C. Tiraby $\cdot$ L. Lichtenstein $\cdot$ J. S. Iacovoni $\cdot$ A. Mairal $\cdot$ D. Langin $(\bowtie)$

Inserm U858, Institut de Médecine Moléculaire de Rangueil, Laboratoire de recherches sur les obésités,

Equipe 4, 1 avenue Jean Poulhès, Box 84225,

31432 Toulouse Cedex 4, France

e-mail: genevieve.tavernier@inserm.fr

e-mail: dominique.langin@inserm.fr

A. Girousse $\cdot$ G. Tavernier $\cdot$ C. Tiraby $\cdot$ L. Lichtenstein $\cdot$

J. S. Iacovoni $\cdot$ A. Mairal $\cdot$ D. Langin

Université Paul Sabatier, Institut Louis Bugnard, IFR150,

Toulouse, France

F. Villarroya

Departament de Bioquimica i Biologia Molecular and Institut de

Biomedicina, Universitat de Barcelona (IBUB),

Barcelona, Spain

F. Villarroya

CIBER Fisiopatologia de la Obesidad y Nutrición,

Instituto de Salud Carlos III,

Barcelona, Spain

D. Langin

CHU de Toulouse, Laboratoire de biochimie,

Institut Fédératif de Biologie de Purpan,

Toulouse, France studied the molecular mechanisms determining human UCP3 levels in skeletal muscle and their regulation by fasting in transgenic mice.

Methods We produced a series of transgenic lines with constructs bearing different putative regulatory regions of the human UCP3 gene, including promoter and intron sequences. UCP 3 mRNA and reporter gene expression and activity were measured in different skeletal muscles and tissues.

Results The profile of expression and the response to fasting and thyroid hormone of human UCP 3 mRNA in transgenic mice with $16 \mathrm{~kb}$ of the human $U C P 3$ gene were similar to that of the endogenous human gene. Various parts of the UCP3 promoter did not confer expression in transgenic lines. Inclusion of intron 1 resulted in an expression profile in skeletal muscle that was identical to that of human UCP3 mRNA. Further dissection of intron 1 revealed that distinct regions were involved in skeletal muscle expression, distribution among fibre types and response to fasting.

Conclusions/interpretation The control of human UCP3 transcription in skeletal muscle is not solely conferred by the promoter, but depends on several cis-acting elements in intron 1, suggesting a complex interplay between the promoter and intronic sequences.

Keywords Diabetes · Fasting · Regulatory sequences . Transgenic $\cdot$ UCP3 $\cdot$ Uncoupling protein
Abbreviations
CAT Chloramphenicol acetyl transferase
MyoD Myogenic differentiation 1 factor
PPAR Peroxisome proliferator-activated receptor
UCP Uncoupling protein 


\section{Introduction}

Uncoupling protein (UCP) 3 is a member of the mitochondrial carrier superfamily with high sequence identity to UCP1 and UCP2 [1-3]. UCP3 is the only member of the family with a tissue distribution preferentially to skeletal muscle in adult humans. Among skeletal muscles, levels of UCP3 vary according to the type of fibres. Higher levels are found in type II fast-twitch glycolytic and glycolyticoxidative fibres than in type I slow-twitch oxidative fibres [4]. The higher levels in type II than in type I fibres may be related to the respective content in mitochondria and associated functions. Glycolytic fibres show limited numbers of mitochondria and therefore limited capacity to oxidise lipid and to fight reactive oxygen species production. UCP3 may thereby contribute to the protection of glycolytic fibres. Accordingly, mice that lack UCP3 show evidence of increased reactive oxygen species production and oxidative damage in skeletal muscle mitochondria [5]. UCP3 may also play a role in the transport of fatty acid anions. In one model, UCP3 would prevent accumulation of non-esterified fatty acids within the mitochondria preventing mitochondrial damage [6]. In a second model, the export of fatty acyl anions would allow fatty acid reactivation by acyl-CoA synthetases to support fatty acid oxidation [7]. A role in the crosstalk between glucose and fatty acid metabolism is suggested by the effect of UCP3 overproduction in human muscle cells. It results in an increase of fatty acid and glucose oxidation, and also in an enhancement of fatty acid-mediated inhibition of glucose oxidation [8]. These data suggest a role for UCP3 in nutrient partitioning. The importance of UCP3 in wholebody metabolism is further suggested by the fact that high overproduction in skeletal muscle protects against fatinduced insulin resistance [9]. Moreover, physiological overproduction of UCP3 in glycolytic muscles of transgenic mice results in mitochondrial uncoupling, resistance to high fat diet-induced obesity and sex specificity regarding insulin sensitivity and whole-body substrate utilisation [10]. In another transgenic model, physiological overexpression of UCP3 conferred protection from fat gain induced by long-term high-fat feeding [11].

$U C P 3$ gene transcription in skeletal muscle is highly regulated. The production of UCP3 is dependent on the level of cellular differentiation. Muscle cells differentiated in vitro produce little if any UCP3. Myoblast transplantation in immunodeficient mice revealed that fully differentiated skeletal muscle cells are required to reach in vivo levels of human UCP3 mRNA expression [12]. Hormonal and metabolic signals modulate UCP3 levels in vivo. Fasting has been shown to induce UCP3 mRNA expression in rodents and humans $[13,14]$. Fatty acids are potential mediators of this effect, which may at least in part depend on the activation of peroxisome proliferator-activated receptor (PPAR) $\alpha$ in skeletal muscle [15]. Thyroid hormones are also strong inducers of UCP3 expression in humans and in mice [14, 16, 17].

The promoter of the human UCP3 gene has been mapped and partly characterised [18, 19]. In muscle cells, the myogenic differentiation 1 factor $(\mathrm{MyoD})$ is required for substantial promoter activity [20]. Upstream of noncanonical elements binding $\mathrm{MyoD}$ is located a functional PPAR and retinoic acid-responsive element. This site also mediates the thyroid hormone effect, indicating that it behaves as a multihormonal responsive element [21, 22]. However, molecular studies on the regulatory elements governing UCP3 production in skeletal muscle and its regulation by hormones and metabolites have so far relied on cells in culture, which produced very low levels of UCP3 and consequently do not constitute optimal models. Investigation of the transcriptional regulation of UCP3 may be relevant to human metabolism, as several reports have highlighted the association between a polymorphism in the UCP3 promoter and both adiposity and type 2 diabetes in human populations [23-25]. To date, no investigation has been carried out to identify the molecular mechanisms responsible in vivo for muscle production of human or murine UCP3. Here, we studied the importance of regulatory sequences in the human $U C P 3$ gene in vivo in transgenic mice.

\section{Methods}

Generation and analysis of transgenic mouse lines Animal protocols were performed in accordance with the INSERM and the Louis Bugnard Institute Animal Care Facility guidelines. DNA from a bacterial artificial chromosome clone (Genbank accession number AF050113) containing the human UCP3 gene (provided by the Institut de Recherches Servier) was amplified by PCR (Expand Long Template PCR System; Roche Diagnostics, Meylan, France) and the following primers: forward 5' GGCCTCCC AAAGTGCTGGGGTTACAGA 3'; reverse 5' TGCACAC CACATGTCTGCTTTCCAAGG 3'. The 16,023 bp (16 kb) fragment was subcloned into the pGEMT easy cloning vector (Promega, Charbonnières, France). A series of human UCP3 promoter and intronic fragments were amplified by PCR. The PCR products were cloned into the promoterless pCAT3 basic (Promega) and the pSKTNLS LacZ (Stratagene, Amsterdam, the Netherlands) plasmids. Transgenesis was performed as previously described in B6D2/F1 mice [26]. Mice were housed three per cage at $20^{\circ} \mathrm{C}$ with a $12 \mathrm{~h}$ light-dark cycle. For fasting challenges, mice were deprived of food for $48 \mathrm{~h}$ with free access to water. For triiodothyronine treatments, animals of the line expressing the $16 \mathrm{~kb}$ human UCP3 transgene were 
acutely treated with triiodothyronine $(50 \mu \mathrm{g} / 100 \mathrm{~g})$ or vehicle and killed $15 \mathrm{~h}$ later. Tissues were dissected out, rapidly frozen in liquid nitrogen and stored at $-80^{\circ} \mathrm{C}$.

Cell culture and transient transfection assays Myoblastic L6 cells were obtained from American Type Culture Collection (Rockville, MD, USA); brown adipocyte-derived cells HIB-1B were a kind gift of B. Spiegelman (Dana Farber Cancer Institute, Boston, MA, USA). Both types of cells were grown in DMEM containing 10\% (vol./vol.) fetal bovine serum and transfection experiments were carried out in cells at $50 \%$ confluence using FuGene6 Transfection Reagent (Roche). Each transfection point was assayed in triplicate and contained $1.5 \mu \mathrm{g}$ of the plasmids $-3.2 \mathrm{~kb} /$ $\mathrm{h} U C P 3$ promLuc or the $-3.2 \mathrm{~kb} / \mathrm{h} U C P 3$ promLuc/intron 1 (where ' $h$ ' stands for 'human' and 'Luc' for 'luciferase') and, when required, $0.3 \mu \mathrm{g}$ of the plasmid mammalian expression vectors for various transcription factors and $3 \mathrm{ng}$ of pRL-CMV (Promega), an expression vector for the sea pansy (Renilla reniformis) luciferase used as an internal transfection control. Cells were incubated for $48 \mathrm{~h}$ after transfection. Luciferase activity elicited by UCP3 promoter constructs was normalised for variation in transfection efficiency using Renilla luciferase as an internal standard.

Quantification of $m R N A$ Mouse tissues were homogenised in RLT buffer (RNA fibrous tissue kit; Qiagen, Courtaboeuf, France) using a rotor-stator. Total RNA from skeletal muscle was extracted using RNeasy kit (Qiagen). Quantitation of mRNA levels was performed by reverse transcription quantitative PCR [27].

Reporter gene assay Choramphenicol acetyl transferase (CAT) and $\beta$-galactosidase activities were determined on tissue homogenates using standard procedures [28]. In cell transfection experiments, firefly luciferase and Renilla luciferase activities were measured in a luminometer
(TD20/20; Turner Designs, Sunnyvale, CA, USA) using a kit (Dual Luciferase Reporter assay system; Promega).

Computer-assisted analysis Genomic regions encompassing the UCP3 loci were extracted from the UCSC Genome database (available at http://genome.ucsc.edu, accessed 9 October 2007) for human, mouse, rat, chimpanzee, rhesus, dog and cow. Human intron 1 and various subsequence fragments were fed in the blastz/Threaded Blockset Aligner pipeline in order to generate alignments to use as a conservation filter in subsequent transcription factor binding site analyses. Jaspar matrices were used in conjunction with the Cis-eLement Over-representation (Clover) search program to look for putative binding sites in the human sequence (http://jaspar.cgb.ki.se, accessed 9 October 2007; the Clover program is available for downloading at http://zlab.bu.edu/ clover). The multi-species alignment was then used to assess the degree of conservation of putative sites (Jaspar PMID: 14681366, Clover PMID: 14988425, TBA PMID: 15060014, UCSC PMID: 18086701). A threshold of 0.95 was set for matrix similarity score to ensure good sensitivity and specificity. Thus, only predicted binding sites with a score over the above threshold were taken into account.

Statistics Values are means \pm SEM. Statistical significance was determined using the non-parametric Mann-Whitney test and the SPSS package, version 11.0 for Windows (SPSS, Chicago, IL, USA).

\section{Results}

The human UCP3 gene is expressed in skeletal muscle of transgenic mice To gain insights into the sequences controlling expression of the human UCP3 gene in vivo, different transgenic mouse lines were created (Fig. 1). Two lines of
Fig. 1 Genomic organisation of human UCP3 and of intronic fragments used in transgenesis. a A $16 \mathrm{~kb}$ region including the promoter region, exons, introns and a short $3^{\prime}$ flanking region. White boxes, non-coding sequences in exons; black boxes, coding regions.

b Fragments from intronic and exonic sequences used in transgenic constructs. c Subdivision of the first intron in fragments named I1 to I4 used in transgenic constructs

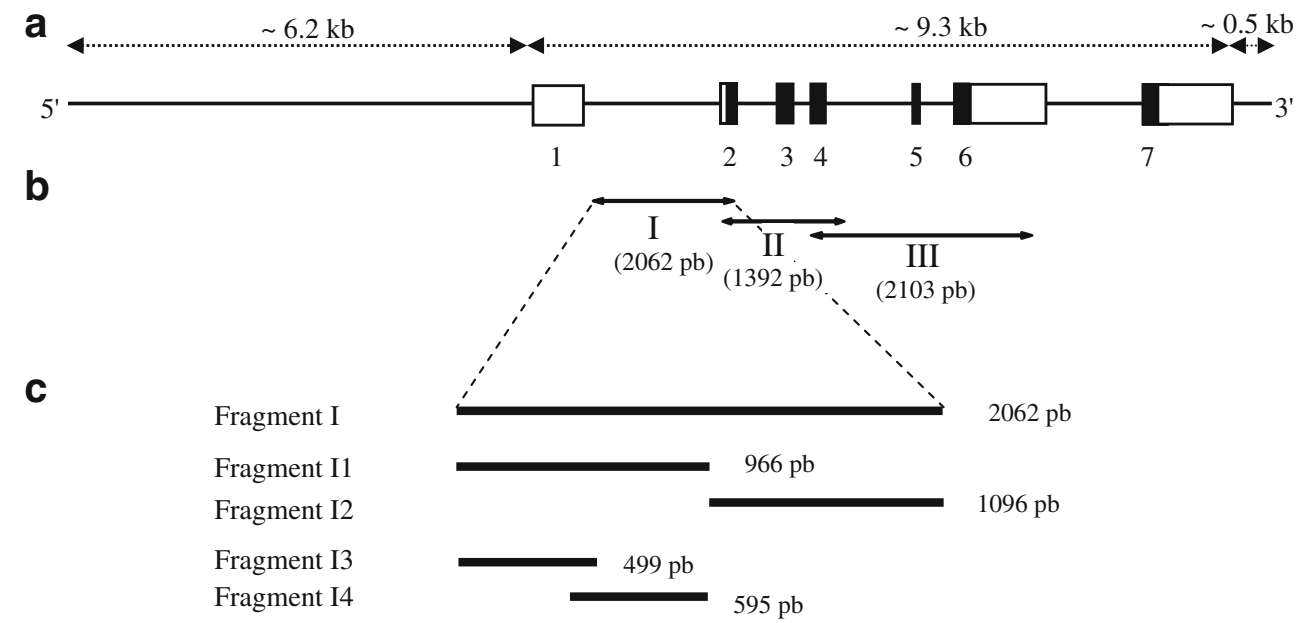


mice were produced with a $16 \mathrm{~kb}$ human $U C P 3$ transgene containing $6.2 \mathrm{~kb}$ of the promoter and the entire exonintron sequences. Human and murine UCP3 mRNA amounts were quantified in various tissues (Fig. 2a-c). In the two transgenic lines, human UCP3 was expressed at higher levels in glycolytic (biceps femoris and gastrocnemius) than in mixed (diaphragm) and oxidative (soleus) muscle. In heart and brown adipose tissue, the level of human UCP3 mRNA was very low. As expected, no human UCP3 mRNA expression was detected in the kidney. Murine $U c p 3$ mRNA expression was also higher in the gastrocnemius and biceps femoris glycolytic muscle than in the oxidative soleus muscle. A noticeable difference from human UCP3 was the substantial expression of mouse Ucp 3 mRNA in heart and brown adipose tissue.

To determine whether the $16 \mathrm{~kb}$ fragment contained the sequences responsible for human UCP3 gene regulation in vivo, transgenic mice were submitted to fasting and treated with thyroid hormone. A twofold induction of human and mouse Ucp 3 mRNA expression was observed during fasting in gastrocnemius muscle (Fig. 3a, c). Acute thyroid hormone treatment also promoted induction of human and mouse Ucp 3 mRNA (Fig. 3b, d). These results demonstrate that the $16 \mathrm{~kb}$ human UCP3 transgene contain the cisacting sequences that mediate the effect of fasting and thyroid hormone.

The promoter region is not sufficient to confer skeletal muscle expression of the human UCP3 gene Next, we determined whether the sequences responsible for skeletal muscle expression of human UCP3 were located in the promoter region. Four promoter-CAT gene constructs were microinjected into mouse oocytes (Table 1). Two to five lines were established for each construct with various portions of the human UCP3 promoter. None of the constructs led to expression of $C A T$ determined both by enzymatic activity and mRNA levels in the hindlimb skeletal muscles as well as in kidney, spleen, white and brown adipose tissues, lung, heart and diaphragm (data not shown). No production of CAT was detected in skeletal

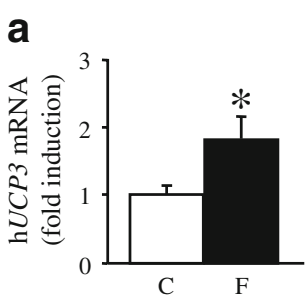

b
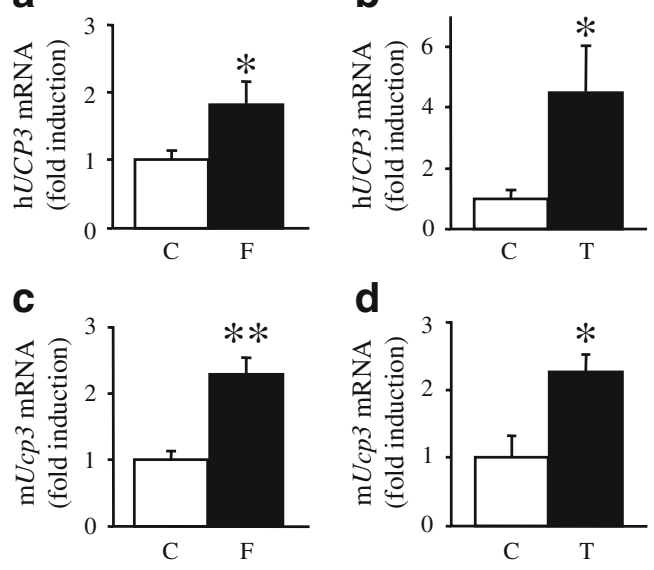

d

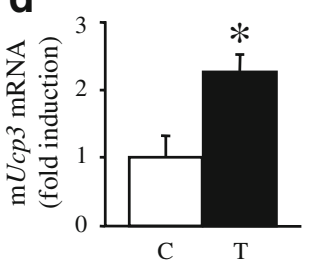

Fig. 3 Effect of $48 \mathrm{~h}$ fasting and acute treatment with thyroid hormone on human and murine UCP3 mRNA in mice expressing the human $U C P 316 \mathrm{~kb}$ transgene. a, c Effect of fasting (F). b, d Effect of thyroid hormone $(\mathrm{T})$. Human UCP3 (hUCP3) and murine Ucp3 (mUcp3) mRNA levels were determined before (C) and after the treatments in gastrocnemius muscle. Results are expressed as fold induction of the mRNA levels quantified in control animals. Data are the means \pm SE $(n=5-9) .{ }^{*} p<0.05,{ }^{* *} p<0.01$

muscle when the $-2.4 \mathrm{~kb} / \mathrm{h} U C P 3$ promCAT mice were studied in conditions of enhanced induction of endogenous $U C P 3$, such as the postnatal period or fasting (data not shown). To determine whether these constructs were functional, transient cotransfection of the $-5.5 \mathrm{~kb} / \mathrm{hUCP3-}$ promCAT with MyoD and retinoic acid receptor expression vectors was performed into muscular L6 cells [20]. A clear induction was observed when MyoD was expressed (6.5 \pm $0.5, p<0.05, n=4)$. Further induction was observed with retinoic acid receptor in the presence of retinoic acid $(9.2 \pm$ $1.1, p<0.05, n=4)$. To exclude an in vivo silencing in the expression of the $C A T$ gene, the $L a c Z$ reporter system was used. Transgenic lines were produced with two promoterLacZ gene constructs. None of them showed $\beta$ galactosidase activity in skeletal muscles. Consequently, promoter sequences are not sufficient to drive skeletal muscle expression of human UCP3 in vivo.

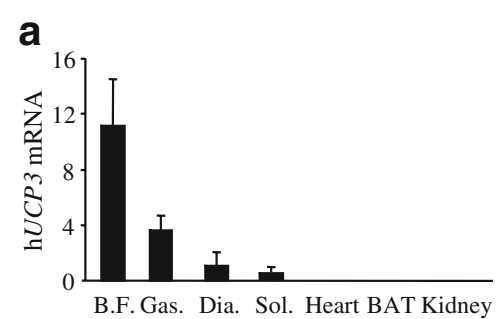

b

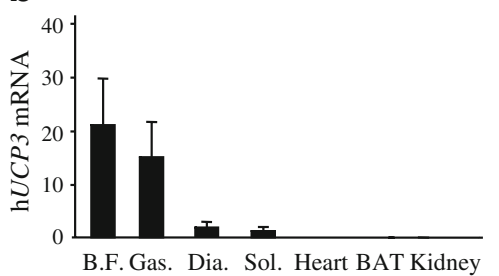

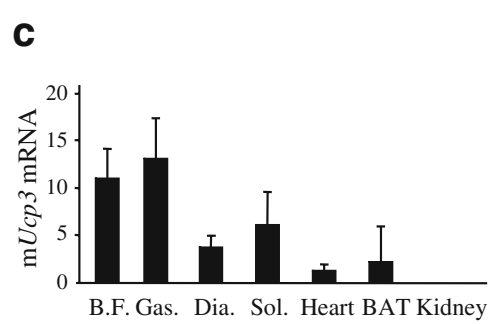

Fig. 2 Expression profile of human and mouse $U C P 3$ mRNA in lines expressing the human UCP3 $16 \mathrm{~kb}$ transgene. a Human UCP3 (hUCP3) mRNA level in transgenic line A. b Human UCP3 mRNA level in transgenic line B. c Mouse $U c p 3$ (mUcp3) mRNA level in the two transgenic lines. Results are expressed as $2^{-\Delta \mathrm{C}_{\mathrm{t}}} \times 10^{9}$. Data are the means \pm SE $(n=5-7)$. BAT, brown adipose tissue; B.F., biceps femoris; Dia., diaphragm; Gas., gastrocnemius; Sol., soleus 
Table 1 Study of the human $U C P 3$ gene promoter in transgenic mice

\begin{tabular}{lllll}
\hline Construct & Founders $(n)$ & Total transgenic lines $(n)$ & Expressing lines $(n)$ & Transgene copy number \\
\hline$-5.5 \mathrm{~kb} / \mathrm{h} U C P 3$ promCAT & 6 & 4 & 0 & $20-50$ \\
$-3.2 \mathrm{~kb} / \mathrm{h} U C P 3$ promCAT & 6 & 5 & 0 & $10-50$ \\
$-2.4 \mathrm{~kb} / \mathrm{h} U C P 3$ promCAT & 3 & 2 & 0 & $20-50$ \\
$-0.4 \mathrm{~kb} / \mathrm{h} U C P 3$ promCAT & 3 & 2 & 0 & $10-50$ \\
$-2.4 \mathrm{~kb} / \mathrm{h} U C P 3$ promLacZ & 3 & 3 & 0 & $20-50$ \\
$-0.7 \mathrm{~kb} / \mathrm{h} U C P 3$ promLacZ & 10 & 10 & 0 & $20-50$ \\
\hline
\end{tabular}

CAT or LacZ activities were determined on hindlimb muscles from three to six mice per transgenic line

The sizes of the promoter region are shown according to the transcriptional start site determined by Acin et al. [19]

Each construct contained 657 bp of exon 1 because several transcriptional start sites have been reported [18, 19, 33]

Intron 1 is essential for UCP3 expression in skeletal muscle As the $16 \mathrm{~kb}$ fragment but not the promoter region confers expression of the transgene, DNA elements downstream of the transcriptional start site are likely to be crucial for human $U C P 3$ expression in skeletal muscle. A new series of CAT constructs was produced containing $3.2 \mathrm{~kb}$ of the promoter and, downstream of the CAT gene, different sequences encompassing the first five introns (Fig. 1b). The transgenic mice were screened for CAT activity in hindlimb muscle (Table 2). The six transgenic lines containing fragments II and III that overlap introns 2 to 5 were negative for $C A T$ expression. However, CAT activity was detected in hindlimb skeletal muscle of two mouse lines bearing the first intron (Fig. 1b). Furthermore, the profile of CAT mRNA expression was determined in the distinct organs (Fig. 4a). CAT mRNA expression was greater in biceps femoris, rectus femoris (another fasttwitch muscle) and gastrocnemius than in diaphragm and soleus muscle. No CAT mRNA expression was detected in heart, brown adipose tissue and kidney. Therefore, the expression of human $U C P 3$ in skeletal muscles is driven by

Table 2 Study of intronic regions of the human $U C P 3$ gene in transgenic mice

\begin{tabular}{lll}
\hline $\begin{array}{l}\text { Name of intronic } \\
\text { fragments }\end{array}$ & $\begin{array}{l}\text { Total transgenic } \\
\text { lines }(n)\end{array}$ & $\begin{array}{l}\text { Expressing } \\
\text { lines }(n)\end{array}$ \\
\hline I & 8 & 2 \\
II & 3 & 0 \\
III & 3 & 0 \\
I1 & 3 & 2 \\
I2 & 3 & 0 \\
I3 & 3 & 0 \\
I4 & 3 & 2 \\
\hline
\end{tabular}

The constructs contain $3.2 \mathrm{~kb}$ of the promoter region, $657 \mathrm{bp}$ of exon 1 , the CAT gene and various intronic regions

The location and size of intronic fragments are shown on Fig. 1

CAT activity was determined on hindlimb muscles from three to six mice per transgenic line regulatory elements within the first intron. To see whether the presence of intron 1 conferred higher expression in muscle cells in vitro, transient transfection experiments were performed in L6 myotubes. Luciferase activity was higher with the construct containing intron 1 than with the construct containing the promoter alone (Fig. 5a). However, the magnitude of increase was much lower than in the in vivo context. No expression was conferred by intron 1 in HIB-B1 cells derived from brown adipose tissue (Fig. 5b). The data show that intron 1 is required for human UCP3 expression in skeletal muscle in vivo.

The first intron was divided into several regions in order to determine more precisely the intronic location of the regulatory elements (Fig. 1c, Table 2). Two transgenic lines containing the $5^{\prime}$ moiety of intron 1 (fragment I1) expressed the transgene in skeletal muscle (Table 2). None of the three lines with the $3^{\prime}$ moiety of the first intron (fragment I2) presented skeletal muscle expression of the reporter gene. The 5' part of intron 1 was cut into two fragments, named I3 and I4, which overlap on about 100 nucleotides (Fig. 1c). While transgenic animals bearing the most $5^{\prime}$ part of intron 1 (499 bp) were negative (fragment I3), two of the three transgenic lines with an internal 595 bp region (fragment I4) showed CAT activity in hindlimb muscle without ectopic expression in liver or spleen (data not shown). This small region of intron 1 contains elements necessary for skeletal muscle expression of human UCP3 in vivo.

The expression pattern among different type of skeletal muscle was determined by measuring CAT mRNA levels in the different positive transgenic lines (Fig. 4b). Intron 1 (fragment I) and its 5 ' moiety (fragment I1) conferred a profile similar to the human UCP3 gene. However, the transgenic mice with the $595 \mathrm{bp}$ internal region of intron 1 (fragment I4) had a profoundly altered pattern with strong expression of CAT mRNA in the diaphragm and soleus. A similar profile was observed at the protein level with CAT enzymatic assay (data not shown). Altogether, the data reveal that skeletal muscle expression of human UCP3 depends on an internal region of intron 1 (fragment I4) but 
a

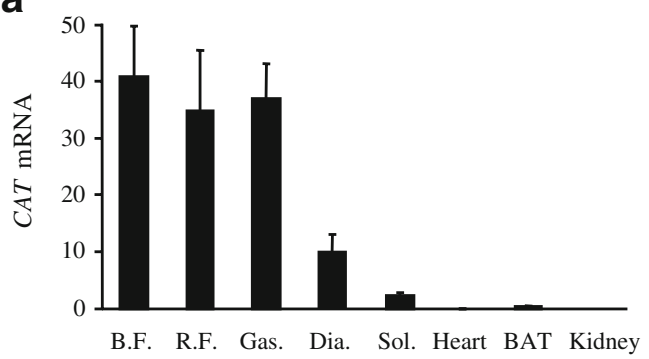

b

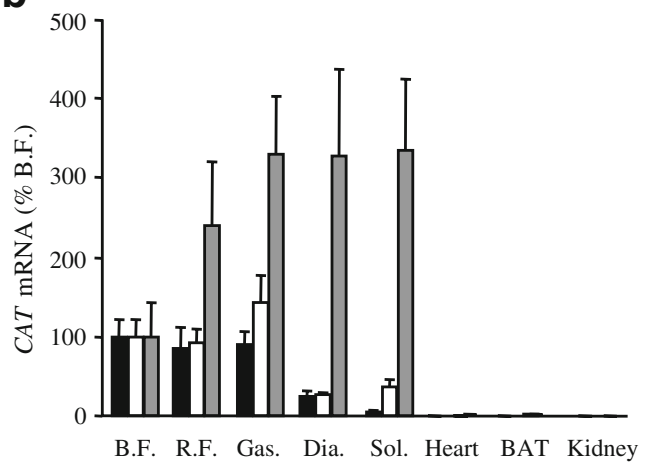

Fig. 4 CAT mRNA expression in tissue from transgenic lines with various fragments of intron 1. a Transgenic mice with the entire intron 1 (fragment I). Results are expressed as $2^{-\Delta C_{t}} \times 10^{9}$. b Comparison of CAT mRNA expression between transgenic lines bearing various portions of intron 1 (location and size of intronic fragments, see Fig. 1). Black bars, animal expressing fragment I of intron; white bars, animal expressing fragment I1 of intron; grey bars, animal expressing fragment I4 of intron. Results are expressed as \% of biceps femoris (B. F.) values. Data are the means \pm SE $(n=5-7)$. BAT, brown adipose tissue; Dia., diaphragm; Gas., gastrocnemius; R.F., rectus femoris; Sol., soleus

that a larger $5^{\prime}$ region (fragment I1) is necessary for proper distribution according to fibre types.

An in silico analysis was next carried out on the entire intron 1 of the human UCP3 gene. Sequence alignments between different species (Homo sapiens, Pan troglodytes, Rhesus macacus, Mus musculus, Rattus norvegicus, Bos taurus and Canis lupus familiaris) revealed important conserved sites located in I3 part, I4 part and at the $3^{\prime}$ end of the intron. Computer-assisted analyses also revealed the existence of several potential sites for transcription response elements. We therefore tested, in L6 cells, the effect of several transcriptional regulators that potentially bind conserved elements, either alone (MyoD, forkhead box [FOX] O1A, FOXO3A, FOXC2, myogenic factor 5 [Myf 5], nuclear respiratory factor $[\mathrm{NRF}] 2 \alpha$, NRF $2 \beta$, v-ets erythroblastosis virus E26 oncogene homologue 2 [ETS]2, PPAR $\alpha$ ), or in combination (MyoD and FOXO3A, FOXC2, $\mathrm{NRF} 2 \alpha, \mathrm{NRF} 2 \beta, \mathrm{ETS} 2$ or PPAR $\alpha$ ), on the $-3.2 \mathrm{~kb} /$ $\mathrm{h} U C P 3$ prom-Luciferase constructs in the absence and the presence of intron 1 . None of these factors led to an increase in luciferase activity associated with the presence of the first intron (data not shown).
Intron 1 is essential for the response to fasting The transgenic mouse lines with expression of the CAT gene in skeletal muscle were submitted to $48 \mathrm{~h}$ fasting to identify the region of intron 1 involved in the response to fasting (Fig. 6). In transgenic mice bearing the entire intron 1 (fragment I), induction of CAT mRNA expression was observed in rectus femoris, gastrocnemius and diaphragm (Fig. 6a). In mice expressing the $5^{\prime}$ moiety of intron 1 (fragment I1) or the internal $595 \mathrm{bp}$ of the first intron (fragment I4), fasting did not induce expression of CAT mRNA in skeletal muscles (Fig. 6b, c). As a control of the effectiveness of fasting, we measured mouse Ucp 3 mRNA levels in the skeletal muscles of mice derived from the different transgenic lines tested. As in wild-type animals, there was an upregulation of murine Ucp3 (data not shown). Therefore, the $3^{\prime}$ part of intron 1 (fragment I2), albeit not necessary for expression in skeletal muscle, is important for the response to fasting.

\section{Discussion}

So far, studies on the molecular mechanisms governing UCP3 regulation have relied on cellular models and in vitro approaches. However, UCP3 expression levels in cultures of skeletal muscle cells are very low compared with in vivo skeletal muscle. This is valid not only for mouse cell lines but also for primary cultures of human skeletal muscle cells [29-31]. Therefore, identification of the regulatory elements critical for total expression of UCP3 at physiological levels is likely to be impossible when relying on transient transfection of promoter-reporter gene constructs in cultured cells. Using a transgenesis approach, our study revealed that the first intron of the human UCP3 gene is essential for proper tissue distribution and that distinct regions are involved in skeletal muscle expression, fibre type specificity and response to fasting (Fig. 7).
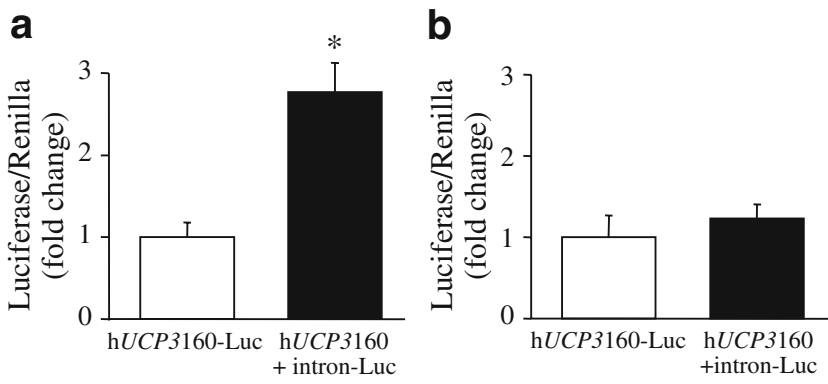

Fig. 5 Luciferase activity of $-3.2 \mathrm{~kb}$ human $U C P 3$ promoter-reporter gene constructs in the presence and absence of intron 1. a Promoter activity in L6 myotubes. b Promoter activity in HIB-IB brown adipocytes. Data are expressed as fold-change relative to $\mathrm{h} U C P 3160-$ Luc activity and are given as means \pm SE $(n=4-6) . * p<0.05$ 
a

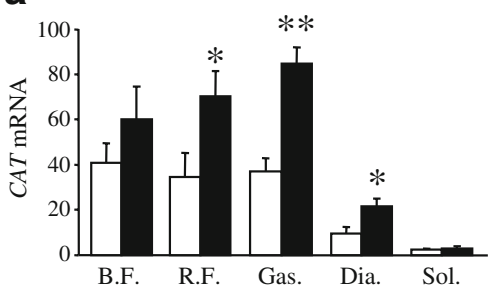

b

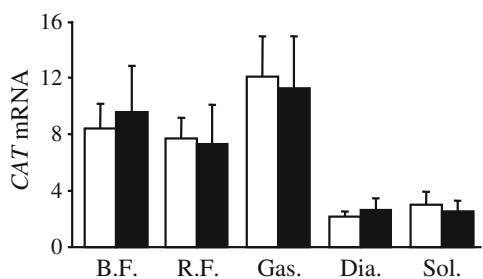

C

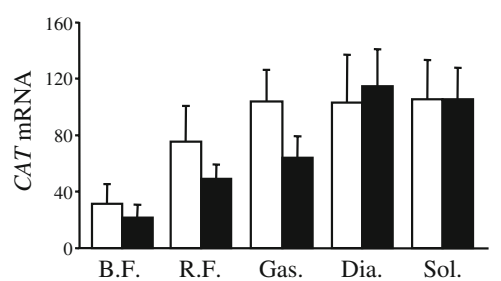

Fig. 6 Effect of $48 \mathrm{~h}$ fasting on CAT mRNA expression in transgenic lines with various fragments of intron 1. a Transgenic mice with fragment I. b Transgenic mice with fragment I1. c Transgenic mice with fragment I4. Black bars, fasted animal; white bars, control fed animal. The location and size of intronic fragments are shown on

A region encompassing $16 \mathrm{~kb}$ of the human $U C P 3$ gene sequence was sufficient to support skeletal muscle expression and regulation in transgenic mice. Higher levels of mouse than human UCP3 transcripts were observed in heart and brown adipose tissue. These data are in accordance with earlier findings suggesting different control of UCP3 expression between rodents and humans. Thus expression of $U C P 3$ mRNA was higher in human than in rat heart [16, 32]. In contrast to rodent brown adipose tissue, where high levels have been reported, little or no expression of UCP3 was detected in human brown adipose tissue $[32,33]$. Moreover, our data with the $16 \mathrm{~kb}$ construct are in agreement with the in vivo distribution of human UCP3 in mice with an $80 \mathrm{~kb}$ transgene encompassing $50 \mathrm{~kb}$ of $5^{\prime}$ flanking region and containing all the intronic regions [22]. Our data on cell lines confirm the specificity of expression of UCP 3 in skeletal muscle cells vs brown adipocytes. Altogether the currently available information suggests that UCP3 shows higher specificity for skeletal muscle expression in humans than in rodents. Considering the profile of expression in the different muscles investigated, the rank order of expression among fibre types, i.e. type IIB $\geq$ type IIA/IIX $>$ type I, in transgenic mice was similar to the endogenous profile described in human skeletal muscle [4]. Moreover, regulation by thyroid hormone and fasting was observed as shown earlier in humans [13, 17]. Therefore, all regulatory sequences crucial for tissue and fibre type distribution, as well as for hormonal and nutritional regulation were present in the $16 \mathrm{~kb}$ transgene. Generation
Fig. 1. CAT mRNA levels were determined in fed and fasted $(48 \mathrm{~h})$ animals. Results are expressed as $2^{-\Delta \mathrm{C}_{\mathrm{t}}}$ values. Data are the means \pm SE $(n=5-7) .{ }^{*} p<0.05,{ }^{* *} p<0.01$. B.F., biceps femoris; Dia., diaphragm; Gas., gastrocnemius; R.F., rectus femoris; Sol., soleus

of numerous transgenic mouse lines revealed that the promoter region is not sufficient for expression of human $U C P 3$ and that intron 1 was indispensable for expression in skeletal muscle and fibre type specificity. The involvement of introns in skeletal muscle gene expression has been shown in vivo in few instances, e.g. for the acetylcholinesterase and dystrophin genes [34, 35]. In both, the enhancer is located in intron 1. Truncation of intron 1 of the human UCP3 gene showed that the $5^{\prime}$ part was necessary for a proper expression profile in the different skeletal muscles. Strikingly, further deletion pointed to an internal part that conferred expression in skeletal muscle, but totally abolished the differences between fibre types. Such a dichotomy makes the human UCP3 gene a unique model to delineate elements critical for skeletal muscle expression from sequences conferring fibre type specificity. The bio-informatic analyses driven on the entire human $U C P 3$ intron 1 revealed that several sequences were conserved among species that could be implicated in transcription factor binding. Conceivably, these transcriptional regulators participate in muscle expression of human $U C P 3$. However, none of the factors expressed transiently in the presence of intron 1 increased expression of the reporter system in L6 myotubes; this could either suggest that the factors and cognate $c i s$-acting motifs tested are not involved in muscle specificity or that in vitro cellular transactivation systems do not support regulatory events occurring in vivo. The data raise the possibility that intron 1 of the human UCP3 gene could reveal new combinations of

Fig. 7 Potential interactions between regions in intron 1 and cis-acting elements in the promoter region of the human $U C P 3$ gene. MREs, uncanonical MyoD responsive elements; PPRE, PPAR responsive element

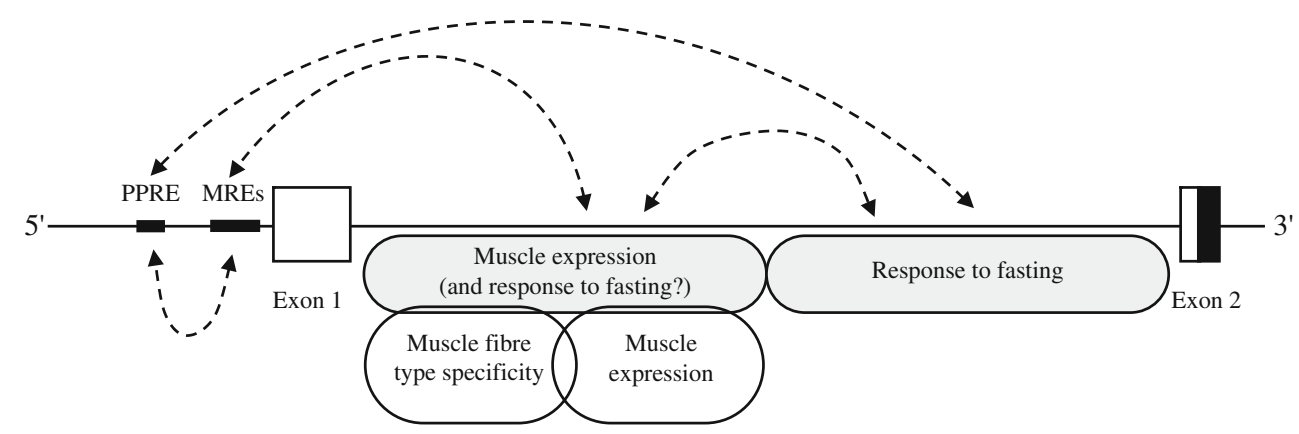


regulatory sequences and transcription factors involved in skeletal muscle expression and fibre type specificity.

Study of the molecular mechanisms controlling gene expression by fasting has received much less attention in skeletal muscle than in liver. The human UCP3 gene is strongly regulated by fasting both in rodents and in humans, an effect potentially mediated by fatty acids $[13$, 36-38]. Interestingly, we observed that the $3^{\prime}$ part of intron 1 , albeit dispensable for muscle expression, was important for the response to fasting. Altogether, the data indicate that different regions of intron 1 are involved in skeletal muscle expression, preferential expression in glycolytic muscles or response to fasting.

Our data shed new light on the control of human UCP3 gene transcription and suggest interactions between cisacting elements in the promoter and different regions of intron 1 (Fig. 7). In vitro studies focusing on the proximal promoter have shown that its activity was dependent on MyoD binding uncanonical elements close to the transcriptional start site [20, 21]. However, the present data clearly show that the $5^{\prime}$ region of intron 1 is needed for skeletal muscle expression in vivo. Therefore, a functional interaction between that region and the proximal promoter could be expected. Furthermore, cooperativity between several elements within that region acts to obtain specific expression in skeletal muscle and proper distribution among muscles with different metabolism. A direct repeat-1 element upstream of the MyoD binding sites mediates activation by PPAR $\alpha$ and $\beta$. Crosstalk between the regulatory elements is suggested by the finding that MyoD is required for activation by a PPAR ligand. Accumulating evidence suggests that fatty acids induce UCP3 gene expression through PPARs [39]. The in vivo analyses described here demonstrate that a critical element in the $3^{\prime}$ part of intron 1 is likely to interact with the PPAR responsive element in the proximal promoter to obtain a full response to fasting. Nevertheless, we cannot exclude the possibility that elements for the response to fasting lie in the $5^{\prime}$ part of the first intron and act in synergy with sequences present in the distal part.

The molecular basis of the preferential expression of human UCP3 in skeletal muscles and its regulation by hormones and nutrients is of interest because skeletal muscles are important in energy homeostasis and because increasing evidence suggests that UCP3 plays a role in this process. Furthermore, UCP3 expression is decreased in type 2 diabetes [40]. This reduction has been shown in prediabetic and diabetic patients [41, 42]. The decrease of $U C P 3$ expression is not attributable to fibre type diminution, since fast-twitch glycolytic fibres, in which $U C P 3$ is preferentially expressed, are increased in muscles of diabetic patients. The relevance of studying regulatory sequences of the human UCP3 gene is furthermore supported by the association between a promoter polymorphism and risk of type 2 diabetes [24]. This study paves the way for future work aimed at dissecting the complex network of interactions between promoter and intronic cisacting elements and at deciphering the mechanisms underlying dysregulation of UCP3 in pathological conditions such as type 2 diabetes.

Acknowledgements This work was supported in part by grants from Inserm (Progres 4P007E), Institut de Recherches Servier and Ministerio de Ciencia e Innovación (SAF2008-01896), Spain. We are very grateful to C. Pujol (Obesity Research Laboratory Inserm U858) and J.-J. Maoret (Molecular Biology Facility of the Louis Bugnard Institute) for expert technical assistance. We also thank Y. Barreira and her team from the Animal Care Facility of the Louis Bugnard Institute.

Duality of interest The authors declare that there no duality of interest associated with this manuscript.

\section{References}

1. Ricquier D, Bouillaud F (2000) The uncoupling protein homologues: UCP1, UCP2, UCP3, StUCP and AtUCP. Biochem J 345:161-179

2. Krauss S, Zhang CY, Lowell BB (2005) The mitochondrial uncoupling-protein homologues. Nat Rev Mol Cell Biol 6:248-261

3. Brand MD, Esteves TC (2005) Physiological functions of the mitochondrial uncoupling proteins UCP2 and UCP3. Cell Metab 2:85-93

4. Hesselink MKC, Keizer HA, Borghouts LB et al (2001) Protein expression of $U C P 3$ differs between human type 1, type $2 \mathrm{a}$, and type $2 \mathrm{~b}$ fibers. FASEB J 15:1071-1073

5. Vidal-Puig AJ, Grujic D, Zhang C-Y et al (2000) Energy metabolism in uncoupling protein 3 gene knockout mice. J Biol Chem 275:16258-16266

6. Schrauwen P, Hoeks J, Hesselink MK (2006) Putative function and physiological relevance of the mitochondrial uncoupling protein-3: involvement in fatty acid metabolism? Prog Lipid Res 45:17-41

7. Bezaire V, Seifert EL, Harper ME (2007) Uncoupling protein-3: clues in an ongoing mitochondrial mystery. FASEB J 21:312-324

8. Garcia-Martinez C, Sibille B, Solanes G et al (2001) Overexpression of UCP3 in cultured human muscle lowers mitochondrial membrane potential, raises ATP/ADP ratio, and favors fatty acid vs glucose oxidation. FASEB J 15:2003-2035

9. Choi CS, Fillmore JJ, Kim JK et al (2007) Overexpression of uncoupling protein 3 in skeletal muscle protects against fatinduced insulin resistance. J Clin Invest 117:1995-2003

10. Tiraby C, Tavernier G, Capel F et al (2007) Resistance to high-fatdiet-induced obesity and sexual dimorphism in the metabolic responses of transgenic mice with moderate uncoupling protein 3 overexpression in glycolytic skeletal muscles. Diabetologia 50:2190-2199

11. Costford SR, Chaudhry SN, Crawford SA, Salkhordeh M, Harper ME (2008) Long-term high-fat feeding induces greater fat storage in mice lacking UCP3. Am J Physiol Endocrinol Metab 295: E1018-E1024

12. Guigal N, Rodriguez M, Cooper RN et al (2002) Uncoupling protein-3 (UCP3) mRNA expression in reconstituted human muscle after myoblast transplantation in RAG2-/-/gamma c/C5immunodeficient mice. J Biol Chem 277:47407-47411 
13. Millet L, Vidal H, Andreelli F et al (1997) Increased uncoupling protein-2 and -3 mRNA expression during fasting in obese and lean humans. J Clin Invest 100:2665-2670

14. Gong DW, He Y, Karas M, Reitman M (1997) Uncoupling protein-3 is a mediator of thermogenesis regulated by thyroid hormone, beta3-adrenergic agonists, and leptin. J Biol Chem 272:24129-24132

15. Pedraza N, Rosell M, Villarroya J et al (2006) Developmental and tissue-specific involvement of peroxisome proliferator-activated receptor-alpha in the control of mouse uncoupling protein-3 gene expression. Endocrinology 147:4695-4704

16. Larkin S, Mull E, Miao W et al (1997) Regulation of the third member of the uncoupling protein family UCP3 by cold and thyroid hormone. Biochem Biophys Res Commun 240:222-227

17. Barbe P, Larrouy D, Boulanger C et al (2001) Triiodothyroninemediated upregulation of $U C P 2$ and $U C P 3$ gene expression in human skeletal muscle without coordinated induction of mitochondrial respiratory chain genes. FASEB J 15:13-15

18. Solanes G, Vidal-Puig A, Grujic D, Flier JS, Lowell BB (1997) The human uncoupling protein-3 gene. Genomic structure, chromosomal localization, and genetic basis for short and long form transcripts. J Biol Chem 272:25433-25436

19. Acín A, Rodriguez M, Rique H, Canet E, Boutin JA, Galizzi J-P (1999) Cloning and characterization of the 5' flanking region of the human uncoupling protein 3 (UCP3) gene. Biochem Biophys Res Commun 258:278-283

20. Solanes G, Pedraza N, Iglesias R, Giralt M, Villarroya F (2000) The human uncoupling protein-3 gene promoter requires $\mathrm{MyoD}$ and is induced by retinoic acid in muscle cells. FASEB J 14:2141-2143

21. Solanes G, Pedraza N, Iglesias R, Giralt M, Villarroya F (2003) Functional relationship between MyoD and peroxisome proliferator-activated receptor-dependent regulatory pathways in the control of the human uncoupling protein-3 gene transcription. Mol Endocrinol 17:1944-1958

22. Solanes G, Pedraza N, Calvo V, Vidal-Puig A, Lowell BB, Villarroya F (2005) Thyroid hormones directly activate the expression of the human and mouse uncoupling protein-3 genes through a thyroid response element in the proximal promoter region. Biochem J 386:505-513

23. Cassell PG, Saker PJ, Huxtable SJ et al (2000) Evidence that single nucleotide polymorphism in the uncoupling protein 3 (UCP3) gene influences fat distribution in women of European and Asian origin. Diabetologia 43:1558-1564

24. Gable DR, Stephens JW, Cooper JA, Miller GJ, Humphries SE (2006) Variation in the UCP2-UCP3 gene cluster predicts the development of type 2 diabetes in healthy middle-aged men. Diabetes 55:1504-1511

25. Otabe S, Clement K, Dina C et al (2000) A genetic variation in the $5^{\prime}$ flanking region of the UCP3 gene is associated with body mass index in humans in interaction with physical activity. Diabetologia 43:245-249

26. Blaise R, Grober J, Rouet P, Tavernier G, Daegelen D, Langin D (1999) Testis expression of hormone-sensitive lipase is conferred by a specific promoter that contains four regions binding testicular nuclear proteins. J Biol Chem 274:9327-9334

27. Mazzucotelli A, Viguerie N, Tiraby C et al (2007) The transcriptional coactivator PGC-1alpha and the nuclear receptor PPARalpha control the expression of glycerol kinase and metabolism genes independently of PPARgamma activation in human white adipocytes. Diabetes 56:2467-2475

28. Ausubel FM, Brent R, Kingston RE et al (1996) Current protocols in molecular biology. Wiley, New York

29. Hwang CS, Lane MD (1999) Up-regulation of uncoupling protein-3 by fatty acid in $\mathrm{C} 2 \mathrm{C} 12$ myotubes. Biochem Biophys Res Commun 258:464-469

30. Nagase I, Yoshida S, Canas X et al (1999) Up-regulation of uncoupling protein 3 by thyroid hormone, peroxisome proliferator-activated receptor ligands and 9-cis retinoic acid in L6 myotubes. FEBS Lett 461:319-322

31. Chevillotte E, Rieusset J, Roques M, Desage M, Vidal H (2001) The regulation of uncoupling protein- 2 gene expression by omega- 6 polyunsaturated fatty acids in human skeletal muscle cells involves multiple pathways, including the nuclear receptor peroxisome proliferator-activated receptor beta. J Biol Chem 276:10853-10860

32. Boss O, Samec S, Paoloni-Giacobino A et al (1997) Uncoupling protein-3: a new member of the mitochondrial carrier family with tissue-specific expression. FEBS Lett 408:39-42

33. Esterbauer H, Oberkofler H, Krempler F, Strosberg AD, Patsch W (2000) The uncoupling protein-3 gene is transcribed from tissuespecific promoters in humans but not in rodents. J Biol Chem 275:36394-36399

34. Chan R, Boudreau-Larivière C, Angus L, Mankal F, Jasmin B (1999) An intronic enhancer containing an N-box motif is required for synapse- and tissue-specific expression of the acetylcholinesterase gene in skeletal muscle fibers. Proc Natl Acad Sci U S A 96:4627-4632

35. Marshall P, Chartrand N, de Repentigny Y et al (2002) Mouse dystrophin enhancer preferentially targets lacZ expression in skeletal and cardiac muscle. Dev Dyn 224:30-38

36. Weigle DS, Selfridge LE, Schwartz MW et al (1998) Elevated free fatty acids induce uncoupling protein 3 expression in muscle. A potential explanation for the effect of fasting. Diabetes 47:298-302

37. Vidal H, Langin D, Andreelli F, Millet L, Larrouy D, Laville M (1999) Lack of skeletal muscle uncoupling protein 2 and 3 mRNA induction during fasting in type-2 diabetic subjects. Am J Physiol 277:E830-E837

38. Khalfallah Y, Fages S, Laville M, Langin D, Vidal H (2000) Regulation of uncoupling protein-2 and uncoupling protein-3 mRNA expression during lipid infusion in human skeletal muscle and subcutaneous adipose tissue. Diabetes 49:25-31

39. Brun S, Carmona MC, Mampel T et al (1999) Activators of peroxisome proliferator-activated receptor-alpha induce the expression of the uncoupling protein- 3 gene in skeletal muscle: a potential mecha- nism for the lipid intake-dependent activation of uncoupling protein-3 gene expression at birth. Diabetes 48:1217-1222

40. Krook A, Digby J, O'Rahilly S, Zierath J, Wallberg-Henriksson H (1998) Uncoupling protein 3 is reduced in skeletal muscle of NIDDM patients. Diabetes 47:1528-1531

41. Schrauwen P, Hesselink MKC, Blaak EE et al (2001) Uncoupling protein 3 content is decreased in skeletal muscle of patients with type 2 diabetes. Diabetes 50:2870-2873

42. Schrauwen P, Mensink M, Schaart G et al (2006) Reduced skeletal muscle uncoupling protein-3 content in prediabetic subjects and type 2 diabetic patients: restoration by rosiglitazone treatment. J Clin Endocrinol Metab 91:1520-1525 